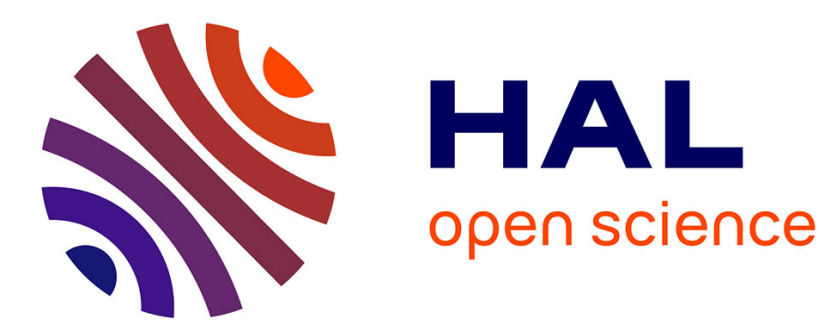

\title{
Tuning methods for the SPIRAL facility
}

\author{
L. Boy, A. Chabert, C. Ricaud, B. Laune
}

\section{To cite this version:}

L. Boy, A. Chabert, C. Ricaud, B. Laune. Tuning methods for the SPIRAL facility. Nuclear Instruments and Methods in Physics Research Section A: Accelerators, Spectrometers, Detectors and Associated Equipment, 1997, 400, pp.1-8. 10.1016/S0168-9002(97)00981-9 . in2p3-00014324

\section{HAL Id: in2p3-00014324 https://hal.in2p3.fr/in2p3-00014324}

Submitted on 21 Dec 1998

HAL is a multi-disciplinary open access archive for the deposit and dissemination of scientific research documents, whether they are published or not. The documents may come from teaching and research institutions in France or abroad, or from public or private research centers.
L'archive ouverte pluridisciplinaire HAL, est destinée au dépôt et à la diffusion de documents scientifiques de niveau recherche, publiés ou non, émanant des établissements d'enseignement et de recherche français ou étrangers, des laboratoires publics ou privés. 


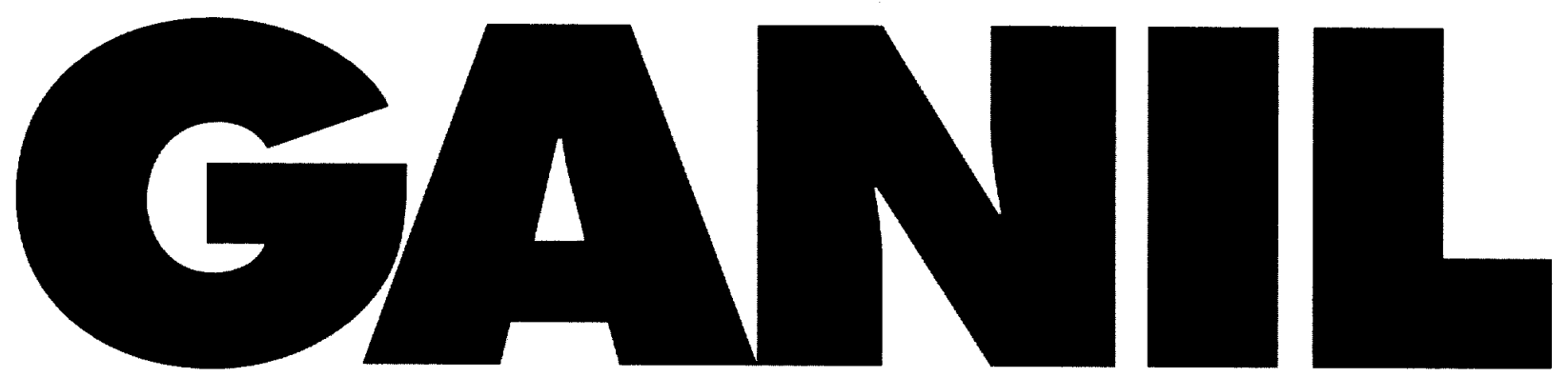

GRAND ACCELERATEUR NATIONAL D'IONS LOURDS - CAEN LABORATOIRE COMMUN IN2P3 (CNRS) - DSM (CEA)

Tuning methods for the SPIRAL facility

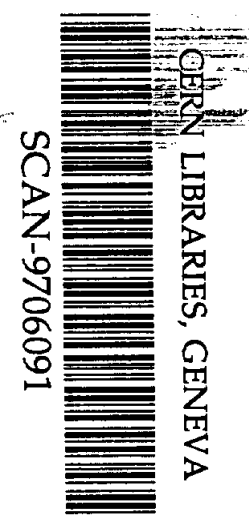

L. Boy, A. Chabert, Ch. Ricaud GANIL (IN2P3/CNRS, DSM/CEA) BP 5027,14021 Caen Cedex, France

B. Launé

Institut de Physique Nucléaire (IN2P3/CNRS), 91406 Orsay Cedex, France

GANIL S 9702

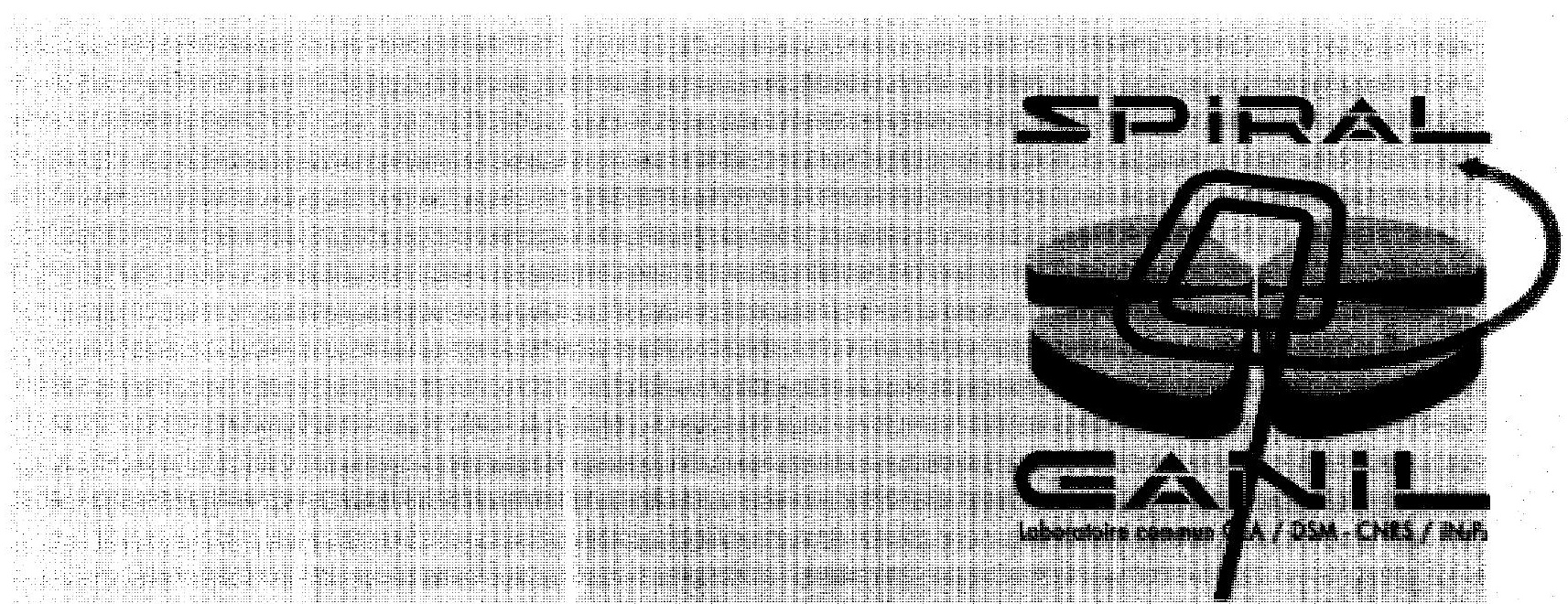




\title{
Tuning methods for the SPIRAL facility
}

\author{
L. Boy, A. Chabert, Ch. Ricaud \\ GANIL (IN2P3/CNRS, DSM/CEA) BP 5027,14021 Caen Cedex, France \\ B. Launé \\ Institut de Physique Nucléaire (IN2P3/CNRS), 91406 Orsay Cedex, France
}

In the SPIRAL facility we will accelerate radioactive ion beams with intensities as small as a few pps mixed with possibly much more abundant unwanted species. Such low currents and the mixing of several species imply special tuning methods and associated diagnostics. The beam lines and the cyclotron will be pretuned with a stable analogue beam close to the radioactive one in term of charge-to-mass ratio. This difference in charge-to-mass ratio implies a shift toward the correct tuning in order to accelerate the required species. Several methods are possible depending on this difference. We can act either on the magnetic field or on the RF frequency or both. Consequences are different for the beam lines and the cyclotron. Constraints on the source extraction voltage and on the injection phase have to be taken into account.

Key words: ISOL, cyclotron, tuning methods.

\section{Introduction}

\subsection{The SPIRAL project}

The SPIRAL project under construction at GANIL [1] will accelerate radioactive ion beams in a large spectrum of energy and intensity. Based on a $k=265$ axially injected-cyclotron, this - ISOL facility uses a thick target and an ECR ion source [2] to produce a wide range of multi-charged radioactive ions.

Figure 1 is a layout of the SPIRAL facility. SPIRAL will use the GANIL heavy ion beams as primary beams : ${ }^{238} \mathrm{U}$ to ${ }^{12} \mathrm{C}$ beams up to 24 and $95 \mathrm{MeV} / \mathrm{u}$ respectively, and the maximum beam power will be increased from the present $400 \mathrm{~W}$ up to $6 \mathrm{~kW}$ for light ions [3]. These improved GANIL primary beams will be fully stopped in a target, producing radioactive atoms by projectile 
or target fragmentation, fusion, fission or spallation. These radioactive atoms will be released from the target at high temperature, and ionized in an ECRIS giving a 0.1 to 0.5 charge-to-mass ratio well adapted to a cyclotron. A Low Energy beam line matches the beams from the ECRIS to the injection into a $3 \mathrm{~m}$ pole diameter compact cyclotron, the average magnetic field ranging from 0.75 to $1.56 \mathrm{~T}$. The extracted energy varies from 1.7 to $6.2 \mathrm{MeV} / \mathrm{u}$ on $\mathrm{RF}$ harmonic modes 5 and 4 and from 4.8 to $25.2 \mathrm{MeV} / u$ on harmonics 3 and 2. After extraction, a Medium Energy beam line drives the beam to the existing experimental areas.

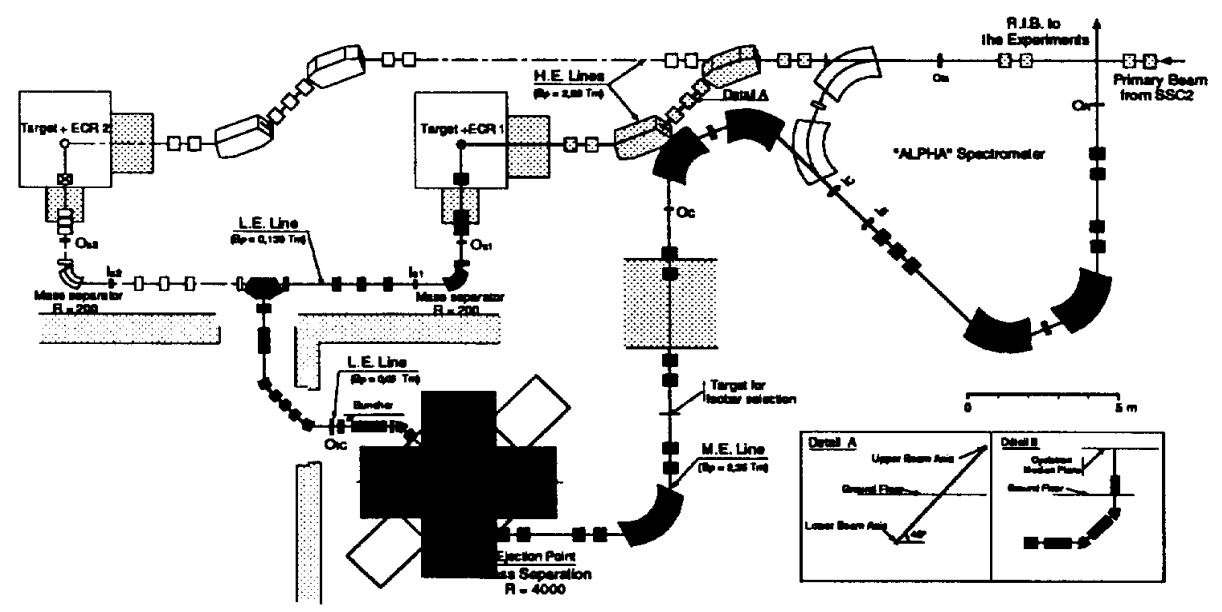

Fig. 1. Layout of the SPIRAL facility.

\subsection{The tuning methods}

The production rate of the radioactive nuclei is usually rather low [4]. Combined with the target diffusion efficiency and the ECRIS ionization efficiency, the expected radioactive beam intensities will be in the range of a few pps to $10^{7-8}$ pps. On the other hand stable pollutants can reach up to $10^{11-12}$ pps. These pollutants can be produced in the target or be issued from the ECRIS chamber and in most cases the beam is a mixture of several species with very different intensities.

As in the other-ISOL facilities a mass analysis is performed in the first part of the injection beam line. It provides a $1 / 200$ mass resolution with an emittance of $80 \pi . m m . m r a d$, but the beam is generally still mixed with other elements up to the injection in the cyclotron. Consequently a direct tuning seems very difficult. Despite of the cyclotron mass resolution $(1 / 2000$ to $1 / 4000$, according to the harmonic mode) pollutants such as isobars or isomeric states may even be extracted. 
To pretune the beam-lines and the cyclotron we will use an analogue stable beam close to the expected one in terms of charge-to-mass ratio. For this pretuning we will use classical diagnostics and it will be performed in parallel with the primary beam tuning thus saving time.

After this pretuning there are two different possibilities depending on the difference of charge-to-mass ratio between the tuning beam and the radioactive beam. If this difference is less than $1-2 * 10^{-4}$ we can not separate them and all we can do is to optimize the intensity of the radioactive beam by monitoring the counting rate after extraction. If this difference is larger, which is generally the case, a shift of the parameters ( $\mathrm{RF}$ frequency, magnetic fields, voltages, or phases) is necessary. In this case, the tuning process can be split into three steps :

(i) tuning of the analogue beam with classical diagnostics,

(ii) shifting of some parameters (depending on the chosen tuning method) according to the theoretical variation,

(iii) optimizing the extracted beam on low intensity diagnostics by refining the injection phase, the cyclotron main field and the acceleration voltage.

The different tuning methods are described in this paper. Results of the preliminary tests of a low intensity radial probe [5] are also presented and compared with the simulation.

\section{Central particle equations}

Usual relativistic notations such as $\beta$ or $\gamma$ are used. For an easy reading the relative variation of the charge-to-mass ratio between the analogue and the expected beam is written:

$$
\epsilon=\frac{d\left(q / m_{0}\right)}{q / m_{0}}
$$

The variables related to the source, the Low Energy beam line, the cyclotron and the Medium Energy beam line are respectively written : "s", "le", "c" and "me". 


\subsection{The beam lines}

\subsubsection{The low energy beam line}

The central energy is given by :

$$
(\gamma-1) m_{0} c^{2}=q e V_{s}
$$

the magnetic rigidity by :

$$
(B \rho)_{l e}=\frac{\beta \gamma m_{0} c}{q e}
$$

and, with $l$ distance between the buncher and the inflector, the injection phase for an RF frequency $f$ by :

$$
\phi_{i n j}=\frac{2 \pi f l}{\beta c}
$$

As in the Low Energy beam line the magnetic field is low $\left(B_{m e} \leq 0.2 T\right)$, a field variation is difficult to realize and we choose to keep the magnetic field unchanged which gives :

$$
\begin{aligned}
d \phi_{i n j} & =\frac{2 \pi f l}{\beta c}\left\{\frac{d f}{f}-\frac{1}{\gamma(\gamma+1)}\left(\epsilon+\frac{d V_{s}}{V_{s}}\right)\right\} \\
\frac{d \rho_{l e}}{\rho_{l e}} & =\frac{1}{(\gamma+1)}\left(\gamma \frac{d V_{s}}{V_{s}}-\epsilon\right)
\end{aligned}
$$

\subsubsection{The medium energy beam line}

Writing the conservation of the magnetic rigidity $\left((B \rho)_{m e}=(B \rho)_{c}\right)$ and assuming the same trajectory in the dipoles $\left(d \rho_{m e}=0\right)$ and that the extraction radius is kept constant $\left(d \rho_{c}=0\right)$ by adjusting the injection phase and/or the cyclotron accelerating voltage $(d V / V \sim-\epsilon)$, we obtain :

$$
\frac{d B_{m e}}{B_{m e}}=\frac{d B_{c}}{B_{c}}
$$

\subsection{The cyclotron}

We choose to keep the trim coil setting unchanged in order to preserve the topology of the field. In addition, due to the hysteresis, the variation of the 
main field must be limited to $10^{-3}$ or less ; this restricts the methods based on cyclotron magnetic field variations to rather low $\epsilon$ values.

Assuming that the trajectories are constant inside the cyclotron $(\delta \rho=0)$, using the isochronism equation ( $h \beta c=2 \pi f \bar{\rho}, h$ harmonic mode) and the previous magnetic rigidity equation, we get :

$$
\frac{\delta B_{c}}{B_{c}}=\gamma^{2} \frac{\delta f}{f}-\epsilon
$$

Since $\gamma$ is a function of the radius this equation is a local equality. As we act on radius independent parameters ( $R F$ frequency, main magnetic field) we need to integrate from the injection to the extraction radius, which gives:

$$
\frac{d B_{c}}{B_{c}} \approx \gamma_{m}^{2} \frac{d f}{f}-\epsilon
$$

The constant $\gamma_{m}$ can have different values from $\gamma_{i}$ to $\gamma_{f}$ (respectively $\gamma$ values at injection and extraction), for instance it can be determined by solving :

$$
\int_{\gamma_{i}}^{\gamma_{f}} \sin (\phi(\gamma)) d \gamma \approx \int_{\gamma_{i}}^{\gamma_{f}} \phi(\gamma) d \gamma=0
$$

which minimizes the energy dispersion of the extracted beam. For a given injection phase different values of $\gamma_{m}$ can be chosen. For example :

- for a zero injection phase :

$$
\gamma_{m}^{2} \approx 1+\frac{2}{3}\left(\gamma_{f}-1\right)
$$

- for a non zero injection phase, with $d W_{t u r n}$ the energy gain per turns, the minimum phase excursion is given by :

$$
\gamma_{m}^{2} \approx \gamma_{f} \text { and } \phi_{i n j} \approx \frac{\pi h m_{o} c^{2}}{3 d W_{t u r n}}\left(\frac{d B_{c}}{B_{c}}+\epsilon\right)\left(\gamma_{f}-1\right)^{2}
$$

In the case of an RF frequency variation, as the trim coil setting is unchanged, the isochronism is not strictly conserved. Figure 2 is an example of the effect of this averaging on the phase law : it shows the tuning of ${ }^{12} \mathrm{~N}$ by RF frequency variation using ${ }^{12} \mathrm{C}$ as the analogue beam. In this case the cyclotron is working at its highest energy and the relativistic effect is maximum. The two possible values for $\gamma_{m}$ mentioned above are used in this simulation : the results are in close agreement with the theoretical ones. The differences seen in the injection phase between the theory and the simulation are a consequence of the "Gaba" [6] effect : the differences in length of the orbits followed in the 
two sectors crossed between accelerating cavities entails a shift in phase on the diagnostic located in the middle of a free valley.

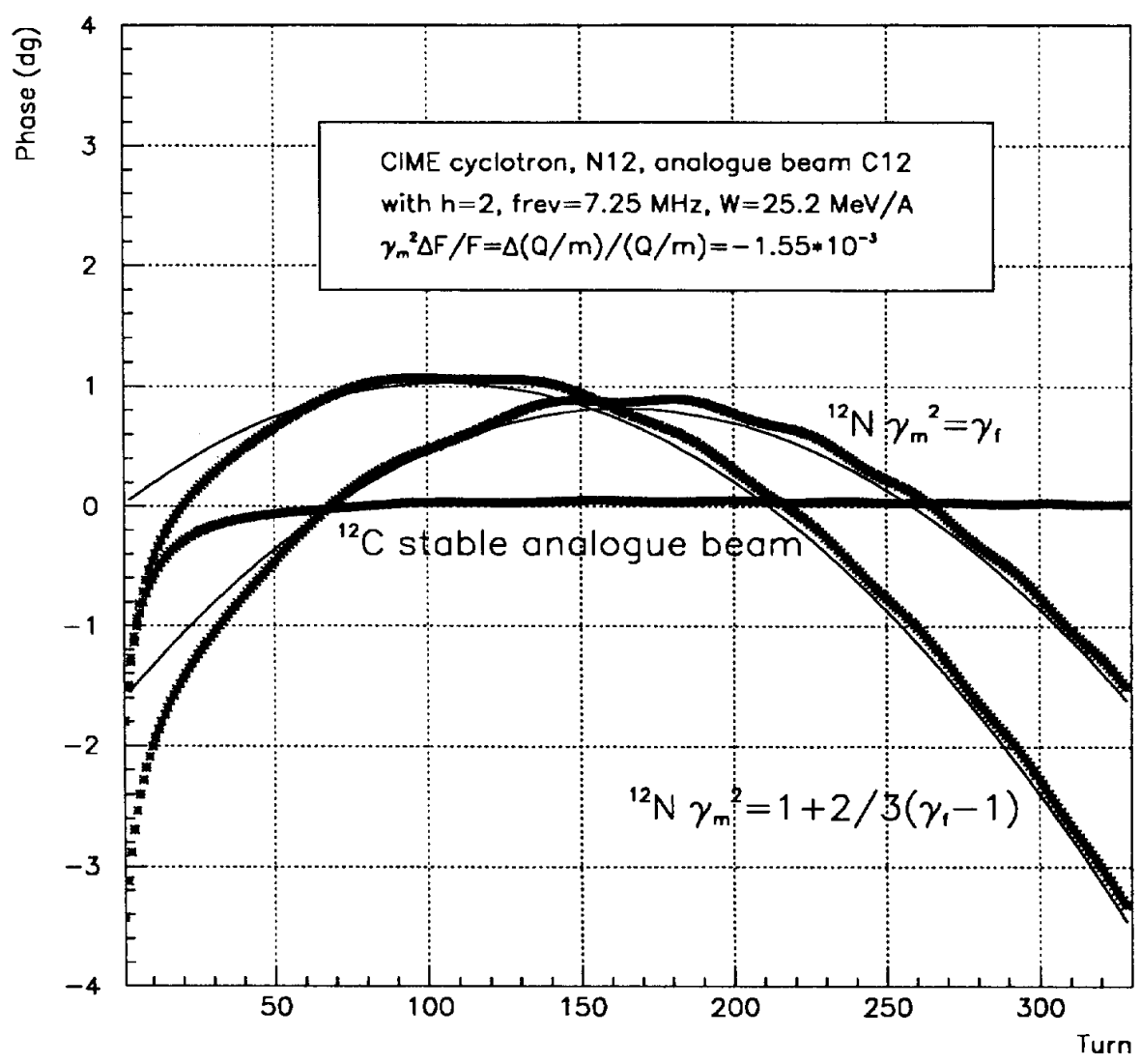

Fig. 2. Phase versus turn number: ${ }^{12} N$ tuning with two possible values of $\gamma_{m}$. Thin lines: the theoretical values, thick lines : the simulation.

\section{Choice of the tuning methods}

As there are five variables we can act on $\left(d B_{c}, d f, d \rho_{l e}, d V_{s}\right.$, and $\left.d \phi_{i n j}\right)$ for only three equations to be fulfilled $((1),(2)$, and (3)) we have to choose between an infinity of solutions. In table 1 we have listed three groups of solutions with one constant parameter ( $\mathrm{A}, \mathrm{B}$, and $\mathrm{C})$, and two particular solutions with two constant parameters ( $D$ and $E$ ). For an easy reading we made the approximation $\gamma=1$ in equations (1) and (2) related to the injection line. 
We need some criteria to make a choice. Obviously, we will try to minimize the number of modifications and to keep constant the least easily reproducible parameters such as the magnetic field. Furthermore, in solutions without frequency variation the isochronism is unchanged and keeping the injection phase constant could be of interest. In the following sections we describe in details the four tuning methods we have chosen.

Table 1

Summary of the solutions with at least one constant parameter.

\begin{tabular}{|c|c|c|c|c|c|}
\hline & $\frac{d B_{c}}{B_{c}}$ & $\frac{d f}{f}$ & $\frac{d \rho_{l \epsilon}}{\rho_{l e}}$ & $\frac{d V_{s}}{V_{s}}$ & $\frac{d \phi_{i n i}}{\phi_{i n i}}$ \\
\hline \hline A & $\gamma_{m}^{2} \frac{d f}{f}-\epsilon$ & free & 0 & $\epsilon$ & $\frac{d f}{f}-\epsilon$ \\
\hline B & $\gamma_{m}^{2} \frac{d f}{f}-\epsilon$ & free & $-\frac{\epsilon}{2}$ & 0 & $\frac{d f}{f}-\frac{\epsilon}{2}$ \\
\hline C & $\gamma_{m}^{2} \frac{d f}{f}-\epsilon$ & free & $\frac{d f}{f}-\epsilon$ & $2 \frac{d f}{f}-\epsilon$ & 0 \\
\hline \hline D & $\left(\gamma_{m}^{2}-1\right) \epsilon$ & $\epsilon$ & 0 & $\epsilon$ & 0 \\
\hline E & $\left(\frac{\gamma_{m}^{2}}{2}-1\right) \epsilon$ & $\frac{\epsilon}{2}$ & $-\frac{\epsilon}{2}$ & 0 & 0 \\
\hline
\end{tabular}

\subsection{Cyclotron magnetic field variation without RF frequency variation}

$$
d f=0 \quad \text { and } \quad \frac{d B_{c}}{B_{c}}=-\epsilon
$$

Solutions (A), (B), and (C) are possible but if we want to keep the variation of the injection phase below one degree, we need $2 * 10^{-5} \leq d \phi_{i n j} / \phi_{i n j} \leq 4 * 10^{-5}$ (depending on the harmonic mode). This is impossible with solutions (A) and (B) in which $d \phi_{i n j} / \phi_{i n j}=-\epsilon$ or $d \phi_{i n j} / \phi_{i n j}=-\epsilon / 2$ as $\epsilon \geq 10^{-4}$, so we chose solution (C). In order to keep the phase constant in equation (1), the injection energy has to be shifted by $d W / W=-d V_{s} / V_{s}=-\epsilon$, but as the injection. radius is small (34 or $45 \mathrm{~mm}$ ) the effect on the injection trajectory is quite negligible.

As seen before, due to hysteresis $\epsilon$ must be small $\left(\epsilon \leq 10^{-3}\right)$. The central trajectories in the beam lines are only slightly modified and the slits of the low energy separator have to be shifted by a few $m m$. The injection phase is also slightly changed by the variation of the trajectory length $\left(2^{\circ}\right.$ for $\epsilon=10^{-3}$ on harmonic 3).

This pure magnetic solution is limited to small values of $\epsilon\left(10^{-4} \leq \epsilon \leq 10^{-3}\right)$. 


\subsection{RF frequency variation without cyclotron field variation}

$$
\frac{d f}{f}=\frac{\epsilon}{\gamma_{m}^{2}} \quad \text { and } \quad d B_{c}=0
$$

The three first solutions are also possible. As $\frac{1}{\gamma_{m}^{2}}-1 \sim 0$ solutions (A) and (C) have two nearly zero parameters, thus eliminating (B) : in solution (A) the phase variation is very small $\left(0.4^{\circ}\right.$ for $\epsilon=10^{-3}$ on harmonic 3$)$ and in solution (C) $d \rho_{l e} / \rho_{l e} \leq 2.6 * 10^{-2} \epsilon$. As the isochronism is not strictly preserved a correction of the injection phase is necessary : this is why solution (A) seems preferable. In this case a shift of the injection energy is also necessary $d W / W=d V_{s} / V_{s}=\epsilon$, but there is no limitation on the value of $\epsilon$ until the resonators are still tuned $\left(\epsilon \leq 10^{-2}\right)$.

\subsection{RF frequency variation with a magnetic correction}

$$
\frac{d f}{f}=\epsilon \quad \text { and } \quad \frac{d B_{c}}{B_{c}}=\left(\gamma_{m}^{2}-1\right) \epsilon
$$

This is solution (D). The relativistic effect $\left(\gamma_{m}\right)$ in the cyclotron is corrected by a small shift of the main field. This solution is very close to the previous one but can be useful for the highest energies. The small phase variation in the case of a pure RF frequency variation (3.2) is balanced by a small magnetic field correction.

\subsection{RF frequency and magnetic variations}

$$
\frac{d f}{f}=\frac{\epsilon}{2} \quad \text { and } \quad \frac{d B_{c}}{B_{c}}=\left(\frac{\gamma_{m}^{2}}{2}-1\right) \epsilon
$$

This is solution (E). Comparing with 3.1 the cyclotron main field variation is divided by two extending the validity of this method up to $\epsilon \leq 2 * 10^{-3}$. There is no source voltage change neither phase change. But as before one can choose not to modify the field in the beam line dipoles at the expense of a trajectory change and consequently a small injection phase shift ( $1^{\circ}$ for $\epsilon=10^{-3}$ on harmonic 3 ). 


\subsection{Summary of the tuning methods}

We have chosen four tuning methods listed in table 2 :

- a method based on a cyclotron magnetic field variation without $R F$ frequency variation for $10^{-4} \leq \epsilon \leq 10^{-3}$

- two methods based on RF frequency variation without limitation on the value of $\epsilon$ :

- an RF frequency variation without cyclotron field variation

- an RF frequency variation with a small cyclotron magnetic field correction

- a mixed method with an RF frequency and a cyclotron magnetic field variation for $10^{-4} \leq \epsilon \leq 2 * 10^{-3}$

The pure magnetic solution is well adapted for small values of $\epsilon\left(\epsilon \leq 10^{-3}\right)$, and the mixed method has almost the same drawback. But if $5 * 10^{-4} \leq \epsilon \leq 10^{-3}$ the tuning beam is lost after a few turns in the cyclotron and we can use a high resolution detector such as the plastic scintillator we have tested (see part 4) to monitor simultaneously the different accelerated elements and to control the magnetic field variations. On the other hand, methods based on RF frequency variations are not limited to small $\epsilon$ values.

Table 2

Summary of the accelerating parameters changes.

\begin{tabular}{|c|c|c|c|c|c|}
\hline \multicolumn{2}{|c|}{ Solutions } & 3.1 & 3.2 & 3.3 & 3.4 \\
\hline \multicolumn{2}{|c|}{ Methods based on } & $d B_{c}$ & $d f$ & $\begin{array}{c}d f \\
+ \text { small } \\
d B_{c}\end{array}$ & $\begin{array}{l}d f \\
+ \\
d B_{c}\end{array}$ \\
\hline \multicolumn{2}{|c|}{ Used if $\epsilon$} & $\leq 10^{-3}$ & $\forall$ & $\forall$ & $\leq 2 * 10^{-3}$ \\
\hline \multicolumn{2}{|c|}{ Isochronism } & unchanged & changed & changed & changed \\
\hline \multirow[t]{2}{*}{ Cyclotron } & $d B_{c} / B_{c}$ & $-\epsilon$ & 0 & $\left(\gamma_{m}^{2}-1\right) \epsilon$ & $\left(\frac{\gamma_{m}^{2}}{2}-1\right) \epsilon$ \\
\hline & $d f / f$ & 0 & $\frac{\epsilon}{\gamma_{m}^{2}}$ & $\epsilon$ & $\frac{\epsilon}{2}$ \\
\hline \multirow{3}{*}{$\begin{array}{c}\text { Low } \\
\text { Energy } \\
\text { beam line }\end{array}$} & $d \phi_{i n j} / \phi_{i n j}$ & $\sim 0$ & $\left(\frac{1}{\gamma_{m}^{2}}-1\right) \epsilon$ & 0 & $\sim 0$ \\
\hline & $d \rho_{l e} / \rho_{l e}$ & $-\epsilon$ & 0 & $\mathbf{0}$ & $-\frac{\epsilon}{2}$ \\
\hline & $d V_{s} / V_{s}$ & $-\epsilon$ & $\epsilon$ & $\epsilon$ & 0 \\
\hline
\end{tabular}




\subsection{Stability}

It is obvious that it will be difficult to control and stabilize relative variations of $10^{-4}$ of the source extraction voltage. If we choose a tuning beam with $\epsilon \leq 5 * 10^{-3}$, it will pass the separator and enter the cyclotron with the radioactive beam. A non destructive capacitive probe will be installed in the last part of the injection line to measure the central phase of this high intensity beam. We will stabilize the injection phase of this beam, and therefore the injection phase of the desired beam, with a feedback on the source voltage. In order to correct the slow variation of the cyclotron magnetic field, we will periodically measure the central phase of the extracted beam with a destructive low intensity diagnostic. Or, if the analogue beam reaches a radius large enough to be monitored on one internal capacitive pick up probes, its central phase can be used for a feedback on the main field.

\section{SPIRAL tuning simulation}

\subsection{Numerical simulation}

Among the possible first beam candidates, ${ }^{34} \mathrm{Ar}^{+8}$ lies in the middle of the cyclotron working diagram : $B_{c}=1.18 \mathrm{~T}, f=12.45 \mathrm{MHz}$ and harmonic 3 . In this case five pollutants are accelerated in the cyclotron but none of them is extracted. The analogue beam is the stable element ${ }^{17} O^{+4}\left(\epsilon=5.3 * 10^{-4}\right)$ which is always present in an ECRIS. The intensity of ${ }^{17} \mathrm{O}^{+4}$ is much greater than the intensity of the other ions. Thus in this example it would be impossible to use low intensity probe on the first meter inside the cyclotron. As the value of $\epsilon$ is rather high and the monitoring impossible on the first meter, we made an RF frequency variation.

Figure 3 displays a numerical simulation made with a 100 particle beam using a field map given by TOSCA. When a beam reaches a $90^{\circ}$ phase before the extraction radius at 1.5 meter, it is decelerated down to the injection radius. The characteristics of the accelerated beams including the maximum radius they reach are summarized in table 3 .

\subsection{Test on the GANIL CSS2}

A low intensity diagnostic based on a scintillator detector was tested on the second GANIL separated sector cyclotron [7]. Other detectors such as semiconductors or micro-channel plates could be used, but the scintillator has been 


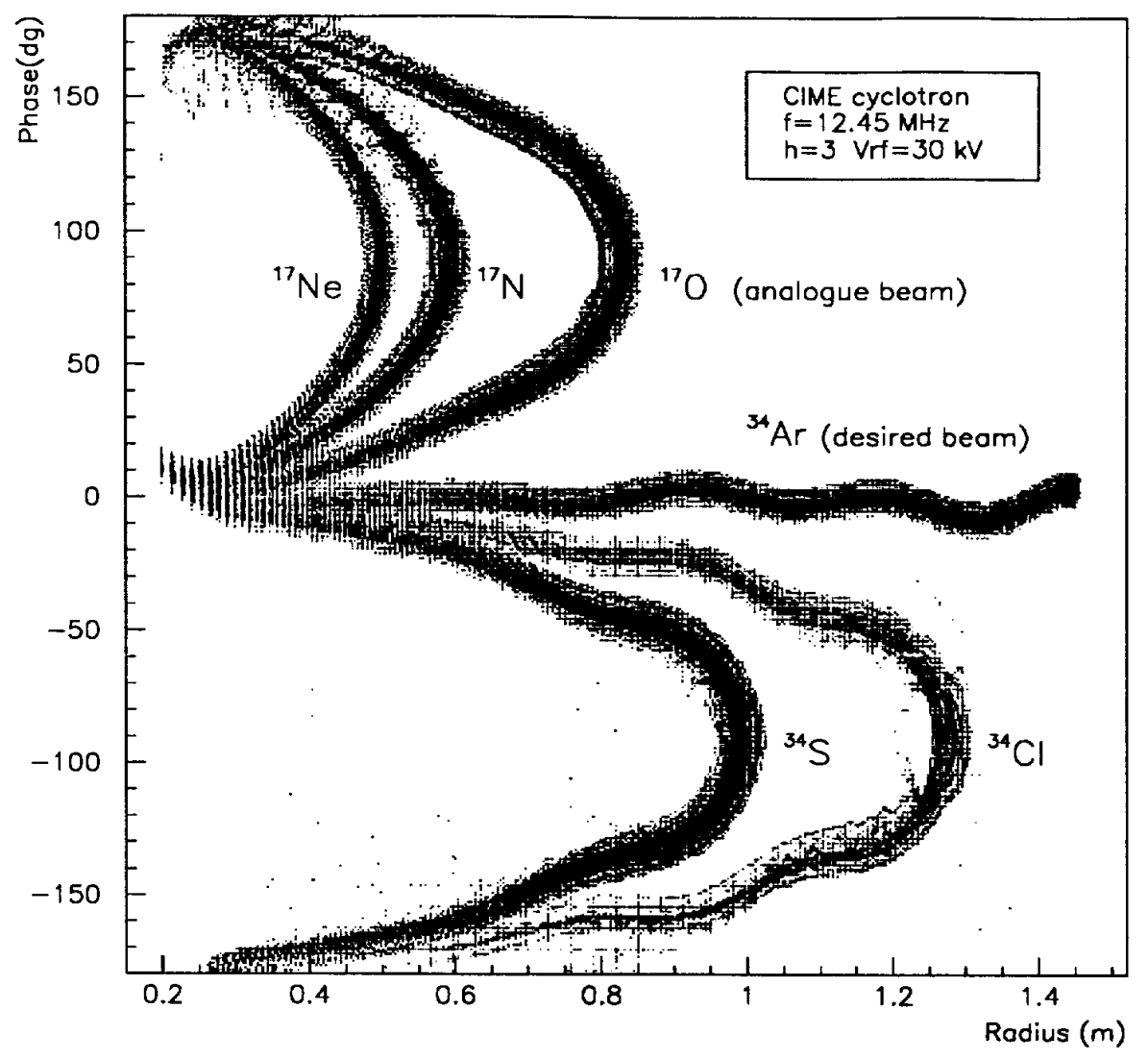

Fig. 3. Phase versus radius simulation of the acceleration of ${ }^{34} A r^{8+}$ in SPIRAL cyclotron in presence of four pollutants.

selected, despite its poor energy resolution, because it is rather robust, cheap and has a good time resolution. This diagnostic allows counting rates up to $10^{4}$ pps (electronic limitation) and the phase of the beam is given (central phase and phase extension) by time measurements with respect to the RF frequency.

The probe is made of a cylindrical $1.8 \mathrm{~cm}$ diameter $4.0 \mathrm{~cm}$ long PILOT-U plastic scintillator mounted on a $8 \mathrm{~m}$ long optical fibre. This fibre is necessary to protect the photo-multiplier from the cyclotron magnetic field.

The experiment was made using a 8 to $60 \mathrm{MeV} / u^{86} \mathrm{Kr}^{+34}$ beam, the intensity being reduced with pepper-pots and slits from $10^{11}$ down to a few pps. The phase versus radius measurement-was made-in flight with $10^{3}$ pps. With such a low intensity it takes about two minutes to measure the isochronism over a two meter scan.

As it is difficult to inject different species in the GANIL SSC we shifted the magnetic field in order to simulate different masses. Figure 4 shows the superposition of two successive phase versus radius measurements with $a \pm 3.5 * 10^{-4}$ main magnetic field variation. 
Table 3

List of the pollutants of an ${ }^{34} A r^{8+}$ beam entering into the cyclotron.

\begin{tabular}{|c|c|c|c|}
\hline Beam & $\epsilon$ & $r_{\max }(m)$ & Stable \\
\hline \hline${ }^{17} N e^{4+}$ & $1.610^{-3}$ & 0.53 & no \\
\hline${ }^{17} N^{4+}$ & $1.110^{-3}$ & 0.62 & no \\
\hline $\begin{array}{c}{ }^{17} O^{4+} \\
\text { (tuning beam) }\end{array}$ & $5.310^{-4}$ & 0.86 & yes \\
\hline $\begin{array}{c}{ }^{34} A r^{8+} \\
(\text { desired beam) }\end{array}$ & 0 & $r_{e j e c}$ & no \\
\hline${ }^{34} \mathrm{Cl}^{8+}$ & $-1.910^{-4}$ & 1.28 & no \\
\hline${ }^{34} S^{8+}$ & $-3.710^{-4}$ & 0.96 & yes \\
\hline
\end{tabular}

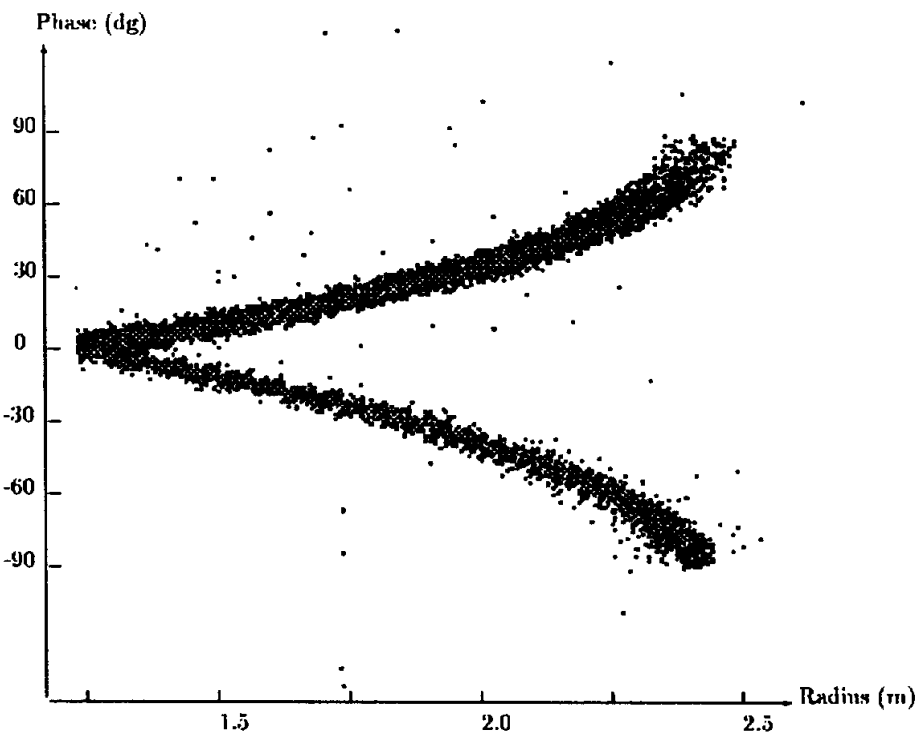

Fig. 4. Superposition of two successive phase versus radius measurements in CSS2 $\left({ }^{86} \mathrm{Kr}\right.$ beam). 


\section{Conclusion}

We reviewed several tuning methods, the one to be chosen for a given case depends on the value of $\epsilon$ and on the number and intensity of the pollutants. Methods based on a frequency variation are simpler and always possible. On the other hand methods based on magnetic variations could be useful for small $\epsilon$ values (a few $10^{-4}$ ) in cases where the absence of an intense pollutant allows a monitoring of the variation. The preferable tuning method is based on frequency variation (3.2) which are not limited to small $\epsilon$ and can always be used. Frequency shifts of $10^{-3}$ are reproducible and will be easily made, without RF cavity short circuit motion. For the highest energies, where $\gamma$ is large, a magnetic correction may be necessary to adjust the injection phase (3.3).

A low intensity radial probe was successfully tested, and new developments are planned for other special diagnostics such as a telescope for identification.

\section{References}

[1] M. Lieuvin and the SPIRAL group. Status of SPIRAL, the radioactive beam project at GANIL. In J. C. CoRnell, editor, Proceedings of the 14th International Conference on Cyclotrons and their Applications, Cape Town, South Africa. World Scientific, October 1995.

[2] L. LeroY et al. ECRIS optimisation for on line production. In M. SEKIGUCHI and T. NAKAGAWA, editors, Proceedings of the 12th International Workshop on ECR Ion Sources, Riken Japan, April 1995.

[3] E. BARON and the GANIL staff. Upgrading the GANIL facilities for high-intensity heavy ion beams. In J. C. ConNell, editor, Proceedings of the 14th International Conference on Cyclotrons and their Applications, Cape Town, South Africa. World Scientific, October 1995.

[4] A.C.C. Villari et al. Radioactive ion beams at SPIRAL. Nuclear Physics A, A588:267-272, 1995.

[5] B. LAuné. The diagnostics system for the SPIRAL R.I.B facility. In J. C. CoRnell, editor, Proceedings of the 14th International Conference on Cyclotrons and their Applications, Cape Town, South Africa. World Scientific, October 1995.

[6] A. Chabert, - G. Gendreau, and P. Lapostolle. Limited energy spread in a ssC. IEEE, 26:2306-2309, April 1979.

[7] L. Boy et al. Cyclotron isochronism tuning by phase measurements with a fast scintillator at very low beam intensity. Submitted to NIM, 1996. 